\title{
Combined kidney-liver perfusion enhances the proliferation effects of hypothermic perfusion on liver grafts via upregulation of IL-6/Stat3 signaling
}

\author{
JIANHUI LI ${ }^{1 *}$, JUNJUN JIA ${ }^{1 *}$, NING HE ${ }^{1 *}$, LI JIANG $^{1}$, HAO YU ${ }^{1}$, HAOYU LI ${ }^{1}$, YIFAN PENG ${ }^{1}$, \\ HAIYANG XIE ${ }^{1-3}$, LIN ZHOU ${ }^{1-3}$ and SHUSEN ZHENG ${ }^{1-3}$ \\ ${ }^{1}$ Department of Hepatobiliary and Pancreatic Surgery, First Affiliated Hospital, School of Medicine, \\ Zhejiang University; ${ }^{2}$ Key Laboratory of Combined Multi-organ Transplantation, ${ }^{3}$ Collaborative Innovation Center for \\ Diagnosis Treatment of Infectious Diseases, Ministry of Public Health, Hangzhou, Zhejiang 310003, P.R. China
}

Received June 20, 2018; Accepted December 19, 2018

DOI: $10.3892 / \mathrm{mmr} .2019 .10379$

\begin{abstract}
A limited number of studies have revealed that adding kidneys to liver perfusion may maintain an improved physiological balance; however, the underlying mechanism remains to be elucidated. The preset study confirmed the protective role of this new model and investigated the underlying mechanisms. Methods: A total of 12 rats were randomly assigned into two groups ( $n=6$ for each group): The kidney-liver perfusion (KL) group and liver perfusion (LP) group. Perfusate samples were collected during the perfusion process for the analysis of $\mathrm{pH}, \mathrm{K}+$ and liver function. Liver tissues were obtained for the evaluation of adenosine triphosphate (ATP), terminal deoxynucleotidyl-transferase-mediated dUTP nick end labelling and immunohistochemistry of Ki67. Cell cycle inhibitors, apoptosis-associated genes and signal transducer and activator of transcription 3 (Stat3) were analyzed using quantitative polymerase chain reaction and western blot analysis. Results: Overall $\mathrm{pH}$ and $\mathrm{K}^{+}$values of the KL group were significantly different from the LP group and more stable; aspartate aminotransferase, alanine transaminase and lactate dehydrogenase levels increased progressively over time in the LP group and were significantly different at different time points compared with pre-perfusion levels and the KL group, which suggested the KL group was superior to the LP group. In addition, KL reduced portal vein resistance and was associated with lower
\end{abstract}

Correspondence to: Professor Shusen Zheng, Department of Hepatobiliary and Pancreatic Surgery, First Affiliated Hospital, School of Medicine, Zhejiang University, 79 Qingchun Road, Hangzhou, Zhejiang 310003, P.R. China

E-mail: shusenzheng@zju.edu.cn

${ }^{*}$ Contributed equally

Key words: kidney-liver perfusion, liver perfusion, hypothermic machine perfusion, liver regeneration, rat
ATP consumption compared with the LP group. Furthermore, liver proliferation was upregulated with the upregulation of the interleukin 6 (IL-6)/Stat3 signaling pathway in KL compared with LP. The present study revealed for the first time that KL and hypothermic machine perfusion demonstrated a more proactive repair capability by maintaining liver regeneration via the upregulation of the IL-6/Stat3 signaling pathway.

\section{Introduction}

Currently, liver transplantation is a first-line treatment for end-stage disease that provides long-term survival. However, with increasing demand, the shortage of donor livers has become a primary concern. Multiple strategies, including marginal grafts [donation following circulatory death (DCD) and extended criteria donor livers (ECD)], have been proposed to overcome the organ shortage. As the demand for transplanted donor organs continues to grow, hypothermic machine perfusion (HMP) has been proposed to be a beneficial alternative to static cold storage (SCS), particularly for marginal grafts.

HMP can be implemented to preserve kidney transplantation grafts. It has been indicated that HMP exhibits improved results compared with SCS. Advances have been also been demonstrated in experimental and clinical liver studies. The majority of studies have demonstrated that HMP could improve or maintain a variety of post-ischemic hepatobiliary parameters, sustain effective liver function and minimize liver damage $(1,2)$.

Different metabolites accumulate during circulation on perfusion, despite the efforts to offset this by use of counteracting substances in order to control the biochemical environment (3,4). Previously, researchers have used membrane dialyzers to filter these metabolites (5). The hypothesis that a filter organ can improve perfusion blood biochemistry has been demonstrated by adding kidney to ex vivo liver perfusion (6). Adding a filter organ to liver circulation helps maintain a better physiological balance in the multi-organ perfusion model, which overcomes the technical challenges of extracorporeal perfusion of multiple organs and the analysis of the response of two organs to the stress states (7). 
Although a number of studies $(6,7)$ have provided certain information regarding kidney liver (KL) perfusion, no studies have implicated KL in HMP and the underlying mechanisms. The present study hypothesized that KL perfusion is superior to liver perfusion alone in protecting liver grafts from ischemic injury. Therefore, in the present study an ex vivo KL perfusion system was used and the initial results were presented to verify the hypothesis and investigate the underlying mechanisms.

\section{Materials and methods}

Animals. In the present study, 12 adult male Sprague Dawley rats (Beijing Vital River Laboratory Animal Technology Co., Ltd., 260-310 g) were used and given free access to water and standard diets and were housed in a 12-h light/dark cycle with a temperature maintained at $20-25^{\circ} \mathrm{C}$ and a relative humidity of $40-60 \%$. All experimental procedures in the present study were approved by the Ethics Committee of the First Affiliated Hospital, College of Medicine, Zhejiang University (2016-374) and were implemented in accordance with the Animal Research: Reporting in vivo experiments guidelines (www. nc3rs.org.uk/arrive-guidelines) and the AVMA euthanasia guidelines 2013.

Experimental design. A total of 12 rats were randomly assigned into two groups ( $\mathrm{n}=6$ for each group). In the KL group, kidneys and livers were preserved by HMP with oxygenated histidine-tryptophanketoglutarate solution perfusate $\left(95 \% \mathrm{O}_{2}\right.$ and $5 \% \mathrm{CO}_{2}$ ) for hypothermic oxygenated perfusion for $6 \mathrm{~h}$. In the LP group, livers were preserved as described in the KL group for $6 \mathrm{~h}$ (Fig. 1).

Organ retrieval and preservation. Rats were anesthetized with $10 \%$ chloral hydrate $(200 \mathrm{mg} / \mathrm{kg}$, Shanghai No. 1 Biochemical and Pharmaceutical Company, Shanghai, China) and none of them exhibited signs of peritonitis. The kidney and liver grafts were retrieved according to the protocols described by Mahboub et al (8) and Kamada et al (9), respectively. After the kidney and liver were isolated, the rats were euthanized by cervical dislocation. The grafts were perfused through the dorsal penile vein with cooled saline containing $25 \mathrm{U} / \mathrm{ml}$ heparin. In the KL group, the renal artery and ureter were cannulated, connected to the oxygenated perfusate $\left(95 \% \mathrm{O}_{2}\right.$ and $5 \% \mathrm{CO}_{2}$ ), recirculated and connected to liver graft via the portal vein (PV; Fig. 2). HMP was performed using a machine perfusion transporter that was constructed as described previously (2), which included a cryostat (DC-1015, Shanghai Bilon Instrument Co., Ltd., Shanghai, China), a four-channel physiology recorder (BL-420S, Chengdu Taimeng Software Co., Ltd., Chengdu, China) and a BT200-2J low flow peristaltic pump (Xi'an Yima Opto-electrical Co., Ltd., Xi'an, China). The perfusion velocity was set at $5 \mathrm{rpm}(3.5 \mathrm{ml} / \mathrm{min})$ and the pressure of the PV was recorded using the computer.

Sample collection. At $0,1,3$ and $6 \mathrm{~h}$ during the perfusion process, $2 \mathrm{ml}$ perfusate samples were collected from the two groups for the analysis of $\mathrm{pH}, \mathrm{K}^{+}$, alanine aminotransferase (ALT), aspartate aminotransferase (AST), lactate dehydrogenase (LDH) and adenosine triphosphate (ATP). Liver tissues were obtained at the end of the preservation and fixed in $10 \%$ neutral formalin at room temperature $\left(18-25^{\circ} \mathrm{C}\right)$ for $24 \mathrm{~h}$ for immunohistochemical analyses. The other liver tissues were stored at $-80^{\circ} \mathrm{C}$ for further experimental analysis.

Immunofluorescence examination and liver function tests. Excised liver specimens were fixed in $4 \%$ paraformaldehyde at room temperature $\left(18-25^{\circ} \mathrm{C}\right)$ for $24 \mathrm{~h}$ prior to being paraffin-embedded and sectioned (thickness, $3 \mu \mathrm{m}$ ). The sections were deparaffinized, hydrated gradually and examined using routine Ki67 immunofluorescence staining as previously described (10). Perfusate samples were collected for ALT, AST, LDH, BUN and CR analysis using the Hitachi 7600 automatic analyzer (Hitachi, Ltd., Tokyo, Japan).

$A T P$ and portal vessel resistance (VR). Portal VR was equal to portal vein pressure/velocity $(\mathrm{ml} / \mathrm{min})$. ATP expression was measured using an ATP kit (Beyotime Institute of Biotechnology, Haimen, China) according to the manufacturer's protocols.

Terminal deoxynucleotidyl-transferase-mediated dUTP nick end labelling (TUNEL) assay. Identification of hepatocyte apoptosis was performed using the TUNEL assay (Roche Diagnostics, Basel, Switzerland) as previously described (11). Briefly, the tissue sections were fixed with paraformaldehyde (4\% in PBS, $\mathrm{pH} 7.4$, freshly prepared) for $20 \mathrm{~min}$ at $15-25^{\circ} \mathrm{C}$. Then they were washed for 30 min with PBS. The slides were incubated in Permeabilization solution $(0.1 \%$ Triton X-100 in $0.1 \%$ sodium citrate, freshly prepared) for $2 \mathrm{~min}$ on ice $\left(4^{\circ} \mathrm{C}\right)$. The slides were rinsed twice with PBS. Then $50 \mu 1$ TUNEL reaction mixture (45 $\mu 1$ TUNEL Label with $5 \mu 1$ TUNEL Enzyme) was added to the sample. The section was incubated for $60 \mathrm{~min}$ at $37^{\circ} \mathrm{C}$ in a humidified chamber in the dark. The slide was then rinsed 3 times with PBS. DAPI $(0.5-10 \mu \mathrm{g} / \mathrm{ml})$ was added and the slide incubated for $10 \mathrm{~min}$ at room temperature in a humidified chamber in the dark. The slide was then rinsed 3 times with PBS. Apoptotic hepatocytes were examined using a fluorescence microscope at a magnification, $\mathrm{x} 200$.

Quantitative polymerase chain reaction ( $q P C R$ ). Total RNA was derived from liver homogenates using TRIzol reagent (Thermo Fisher Scientific, Inc., Waltham, MA, USA). qPCR was performed using a SYBR Green PCR kit (Takara Bio, Inc., Otsu, Japan). The gene expression levels of Caspase 3, B-cell lymphoma-2-associated X (Bax), P21 and P27 were quantified using a 7900 Fast Real-Time PCR instrument. The following primers were used: Caspase 3, forward, 5'-CGG TATTGAGACAGACAGTGGA-3' and reverse, 5'-CGCAAA GTGACTGGATGAAC-3'; Bax, forward, 5'-TTTGCTACA GGGTTTCATCCA-3' and reverse, 5'-TGTCCAGTTCAT CGCCAAT3-3'; P21, forward 5'-AAAGTATGCCGTCGT CTGTTC-3' and reverse, 5'-AAGTCAAAGTTCCACCGT TCTC-3'; P27, forward, 5'-ACGGAGCAGAACCCAACT-3' and reverse, 5'-ACCATTAGCGTGTCCAGG-3'; and $\beta$-actin, forward, 5'-ACGGTCAGGTCATCACTATCG-3' and reverse, 5'-GAGGTCTTTACGGATGTCAACG-3'. Gene expression was quantified using the following conditions: 1 cycle of $95^{\circ} \mathrm{C}$ for $30 \mathrm{sec} ; 40$ cycles of $95^{\circ} \mathrm{C}$ for $5 \mathrm{sec}$; and 1 cycle of $60^{\circ} \mathrm{C}$ for $30 \mathrm{sec}$. The method of quantification was performed as previously described (12). 

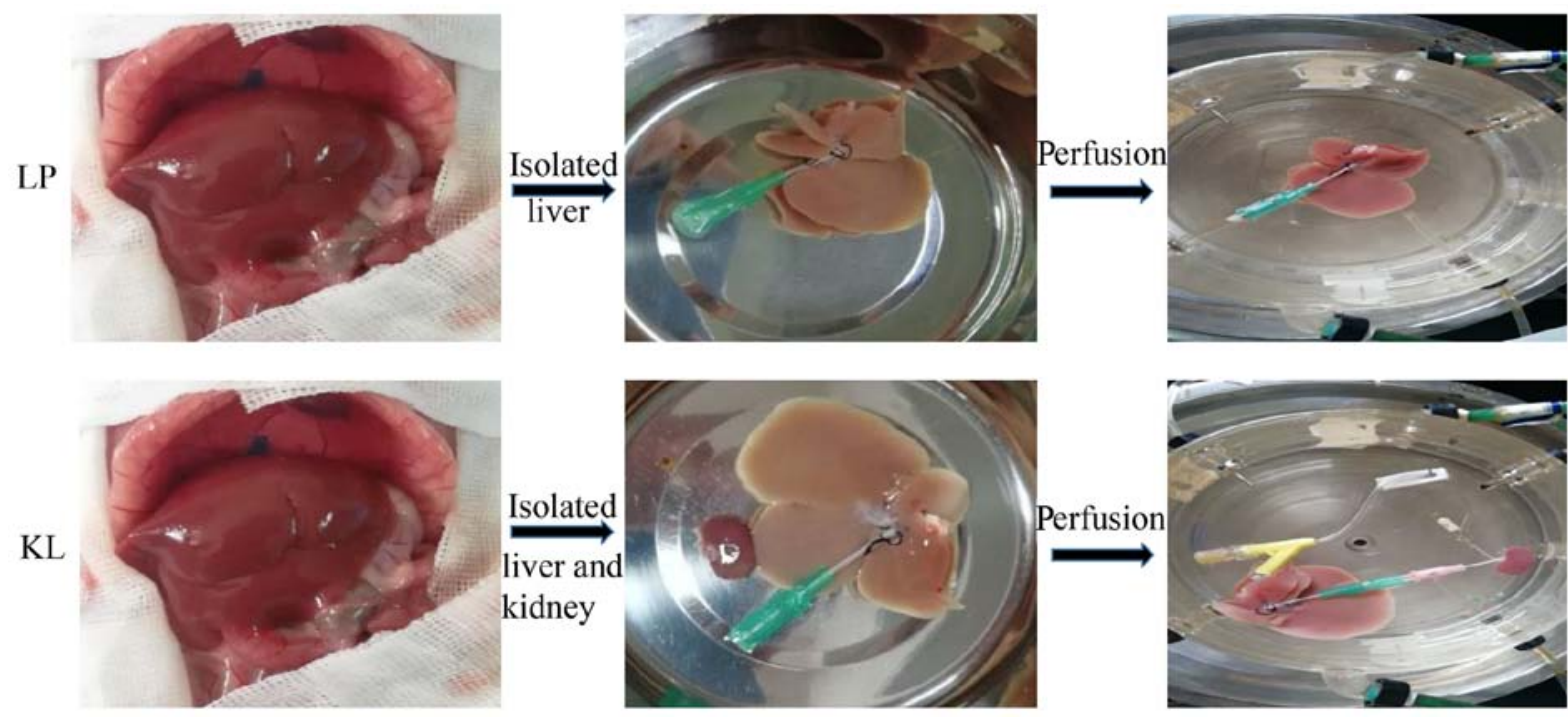

Figure 1. LP and KL model of the study. LP, Liver perfusion; KL, Kidney liver perfusion.

IL-6, hepatocyte growth factor (HGF) and tumor necrosis factor- $\alpha(T N F-\alpha)$. Liver lysates were quantified. Protein concentrations of IL- 6 , HGF and TNF- $\alpha$ were determined using the IL-6 (R6000B), HGF (MHG00) and TNF- $\alpha$ (DY510) ELISA assay kits (R\&D systems, Inc., Minneapolis, MN, USA) according to the manufacturer's protocol.

Western blot analysis. Liver tissues were homogenized in radioimmunoprecipitation assay buffer (Beyotime Institute of Biotechnology). Following centrifugation (at 15,000 x g for $15 \mathrm{~min}$ at $4^{\circ} \mathrm{C}$ ), protein concentrations were quantified using a bicinchoninic acid (BCA) protein assay kit (Thermo Fisher Scientific, Inc.). Proteins $(25 \mu \mathrm{g})$ were separated by $10 \%$ gel electrophoresis and transferred onto nitrocellulose membranes. Following blocking with skimmed milk (232100; BD Biosciences, Franklin Lakes, NJ, USA) at room temperature $\left(18-25^{\circ} \mathrm{C}\right)$ for $2 \mathrm{~h}$, membranes were incubated with primary antibodies at $4^{\circ} \mathrm{C}$ overnight under shaking conditions. The membranes were incubated with cleaved caspase-3 (1:2,000; 9661; Cell Signaling Technology, Inc., Danvers, MA, USA), caspase-3 (1:1,000; 9662; Cell Signaling Technology, Inc.), Bcl-2 (1:500; ab32124; Abcam, Cambridge, MA, USA), Bax (1:500; ab32503, Abcam), phosphorylated (p)-stat3 (1:2,000; 9145; Cell Signaling Technology, Inc.), total (t)-Stat3 (1:1,000; 9139; Cell Signaling Technology, Inc.) and $\beta$-actin $(1: 1,000$; 4970; Cell Signaling Technology, Inc.) primary antibodies. Following this, the blots were washed in TBS-T and incubated with appropriate horseradish peroxidase (HRP)-linked anti-mouse (1:4,000; ab6728; Abcam) or anti-rabbit $(1: 4,000$; ab6721; Abcam) secondary antibodies for $1 \mathrm{~h}$ at room temperature. Then these bands were visualized by chemiluminescence using an enhanced chemiluminescence kit (Pierce; Thermo Fisher Scientific, Inc.). Image J (National Institutes of Health, Bethesda, MD, USA; version 1.48) was used for semi quantitative analysis.

Statistical analysis. Statistical analysis of the data was performed using SPSS version 20.0 (IBM Corp., Armonk, NY, USA). A total of 6 animals each group were used for the experimental and experimental repeats 3 times for each animal. The data were represented as the mean \pm standard error of the mean. Data was analyzed using the one-way analysis of variance followed by the least squares difference or Dunnett's test for multiple comparisons. $\mathrm{P}<0.05$ was considered to indicate a statistically significant difference.

\section{Results}

Effects of $K L$ on $p H$ and $K^{+}$. Overall $\mathrm{pH}$ values of the KL group were significantly different from the LP group and more stable $(\mathrm{P}<0.05)$. The difference resulted from a drop in the LP group at $6 \mathrm{~h}$ of perfusion compared with pre-perfusion levels and the KL group. Such a drop was not identified in the KL group, with no modifications during the perfusion (Fig. 3A). The same trend was indicated regarding $\mathrm{K}^{+}$values (Fig. 3B).

Effects of KL on liver function. ALT levels increased progressively over time in the LP group. Levels were significantly different at 1, 3 and $6 \mathrm{~h}$ compared with pre-perfusion levels and with the KL group at the same time points $(\mathrm{P}<0.05$; Fig. 3C). AST levels also increased progressively over time in the LP group. The levels were significantly different at $6 \mathrm{~h}$ compared with pre-perfusion levels and with the KL group at 1 and $6 \mathrm{~h}(\mathrm{P}<0.05$; Fig. 3D). LDH exhibited the same trend as ALT levels (Fig. 3E).

Effects of KL on VR and ATP. The mean portal pressure of the LP and KL groups was 5 and $4.1 \mathrm{mmHg}$, respectively (Fig. 4A). Portal VR levels between the two groups was indicated in Fig. 4B. The KL group exhibited a significantly lower VR compared with the LP group $(\mathrm{P}<0.05)$. ATP levels between the two groups are presented in Fig. 4C and the higher ATP level was indicated in the KL group compared with the LP group $(\mathrm{P}<0.05)$.

Effects of KL on hepatocyte apoptosis and proliferation. No significant difference was indicated in the number of the 


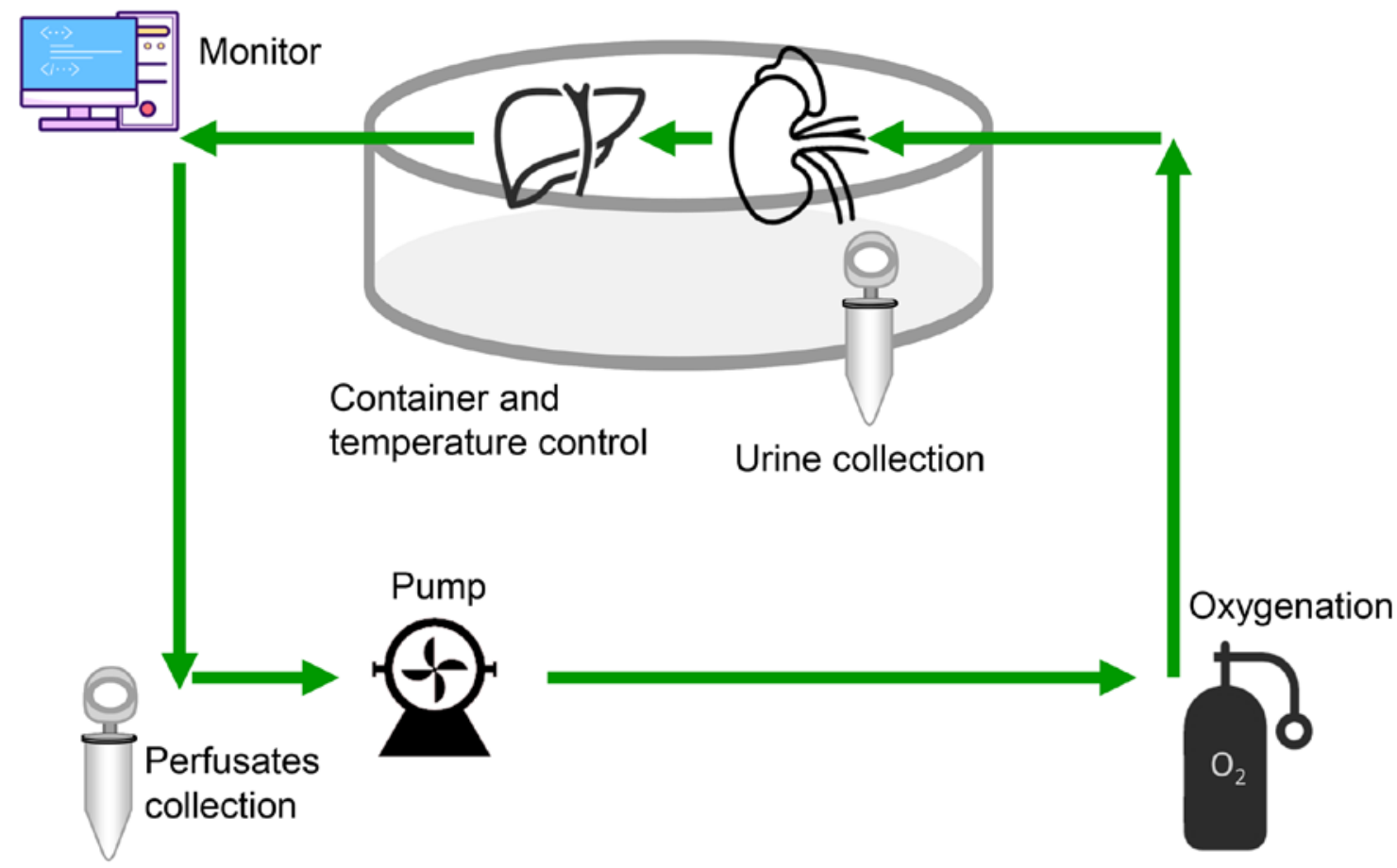

Figure 2. Schematic diagram of kidney-liver hypothermic machine perfusion. In this study, single vessel (portal vein) liver perfusion was performed and urine was collected. No significant results were indicated.

A

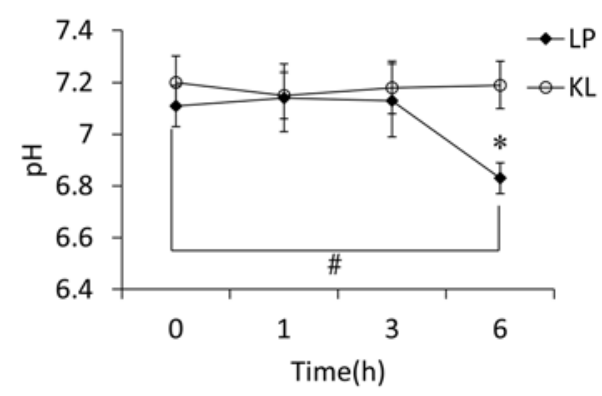

B

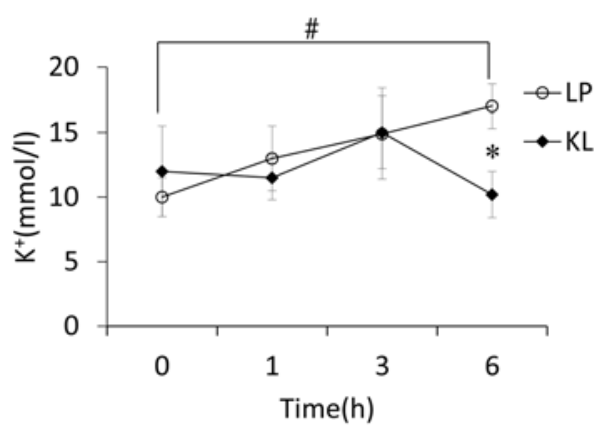

C

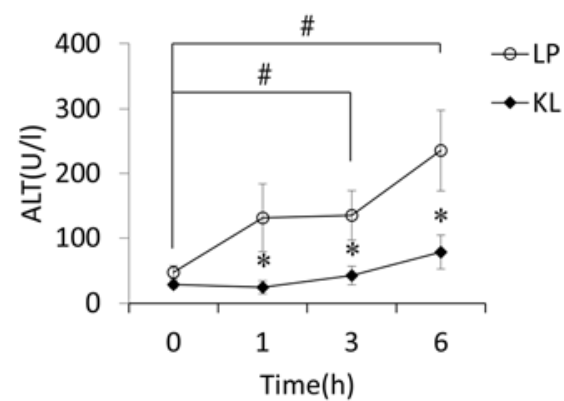

D

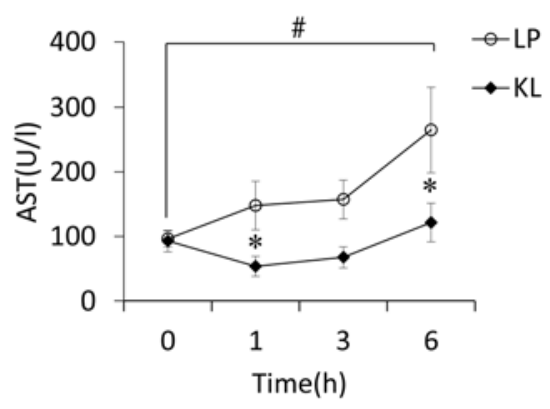

E

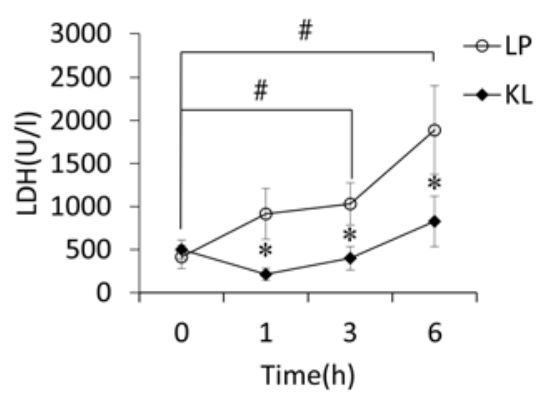

Figure 3. $\mathrm{pH}, \mathrm{K}^{+}$and liver function tests are performed over time. The level of (A) pH, (B) $\mathrm{K}^{+}$, (C) ALT, (D) AST and (E) LDH in KL and LP groups over time. Circles or boxes represented the mean values, whereas bars indicated the mean \pm standard error of mean. ${ }^{*} \mathrm{P}<0.05$ vs. LP group at the same time point; ${ }^{*} \mathrm{P}<0.05$ vs. pre-perfusion levels $(0 \mathrm{~h})$. LP, liver perfusion; KL, kidney liver perfusion; LDH, lactate dehydrogenase; AST, aspartate aminotransferase; ALT, alanine transaminase.

TUNEL ${ }^{+}$hepatocytes in the KL and LP groups (Fig. 5A). Similarly, the same trend was identified in the mRNA (Fig. 5B) and protein expression (Fig. 5C) levels of apoptosis-associated markers (13). Notably, the percentage of Ki67 hepatocytes was increased in the KL group compared with the LP group (Fig. 6A). The mRNA expression levels of P21 (Fig. 6B) and P27 (Fig. 6C) were also measured, which were significantly reduced in the KL group compared with the LP group $(\mathrm{P}<0.05)$. 

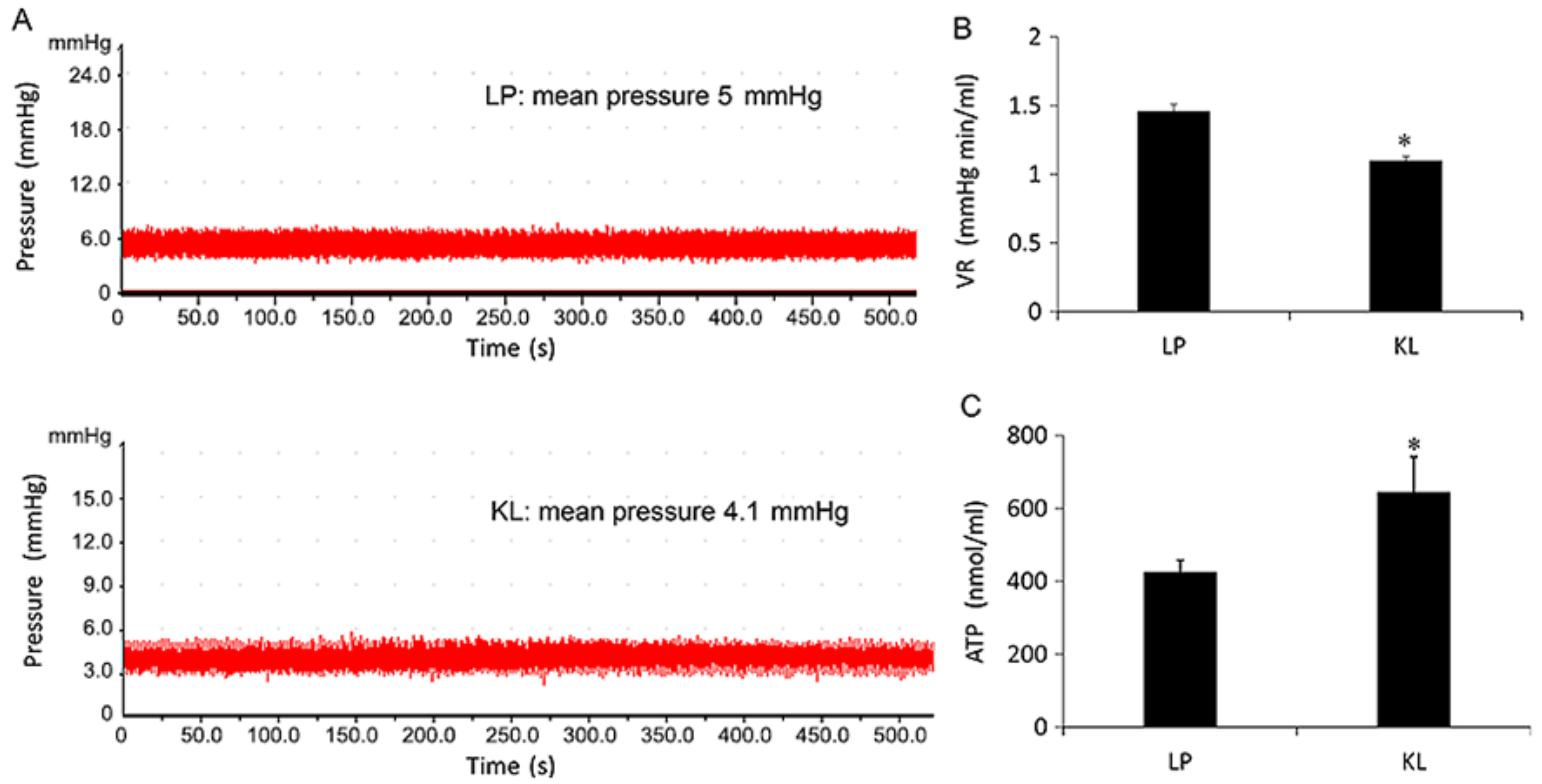

Figure 4. Portal vein resistance and ATP expression in KL and LP groups. (A) The classical pressure record during perfusion from each subgroup. (B) The results of statistical analysis. (C) ATP levels in the KL and LP groups. Data represent mean \pm standard error of the mean. ${ }^{*} \mathrm{P}<0.05$ vs. LP group. ATP, adenosine triphosphate; LP, liver perfusion; KL, kidney liver perfusion; VR, vessel resistance.

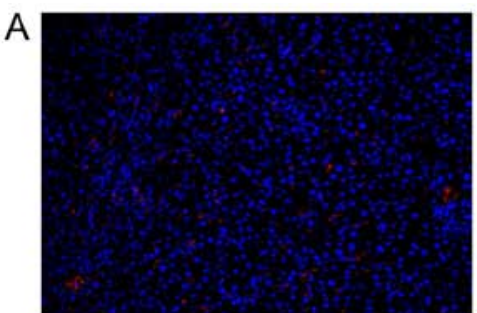

LP

B
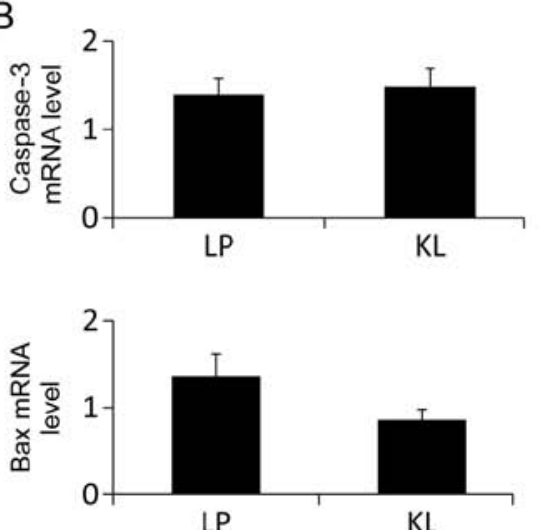

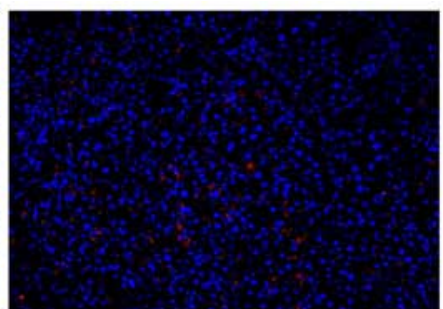

KL

C

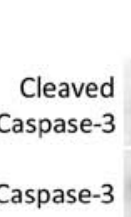

LP

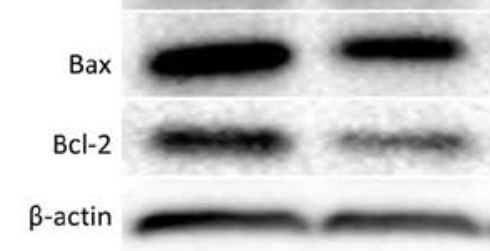

$\mathrm{KL}$
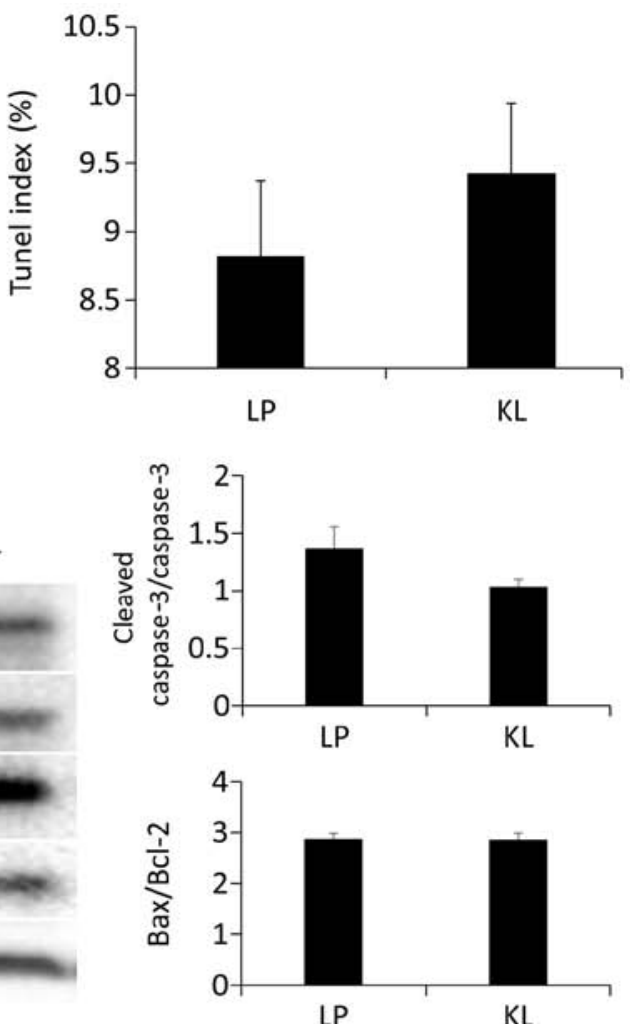

Figure 5. Effects of KL on hepatocyte apoptosis. (A) Identification of hepatocyte apoptosis was conducted by TUNEL assay (n=6, original magnification, x200). The percentage of TUNEL-labeled nuclei was quantified. (B) Quantitative polymerase chain reaction and (C) western blot analysis of apoptosis. The quantitative data of cleaved caspase-3/caspase- 3 and Bax/Bcl-2 are indicated. Data represent the mean \pm standard error of the mean. $\mathrm{LP}$, liver perfusion; KL, kidney liver perfusion; TUNEL, terminal deoxynucleotidyl-transferase-mediated dUTP nick end labelling; B-cell lymphoma-2-associated X, Bax.

Effects of KL on hepatocyte proliferation signaling. IL-6, $\mathrm{HGF}$ and TNF- $\alpha$ levels were examined in liver tissue lysates. The IL-6 levels were significantly increased in the KL group compared with the LP group $(\mathrm{P}<0.05$; Fig. 7A). However, no significant changes in the HGF and TNF- $\alpha$ levels were indicated between the KL and LP groups (Fig. 7B and C). Similarly, the same trend was identified in the protein levels of p-Stat3/t-Stat3 (Fig. 7D). Furthermore, the present results indicated that there was no damage to the kidneys of the KL group compared with the LP group (Fig. 8). 


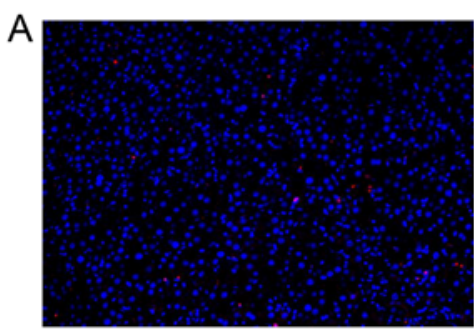

LP

B

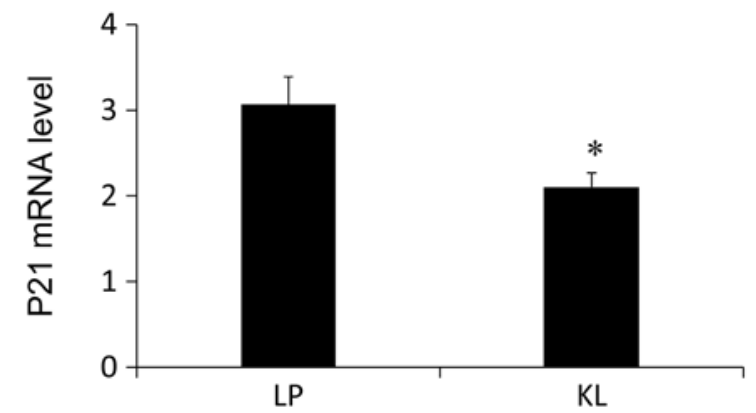

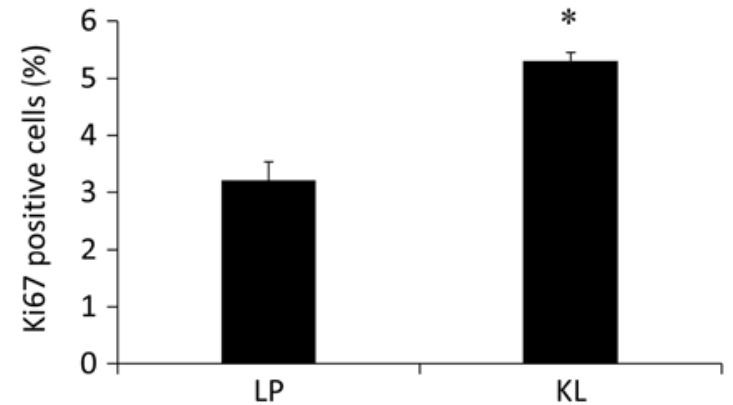

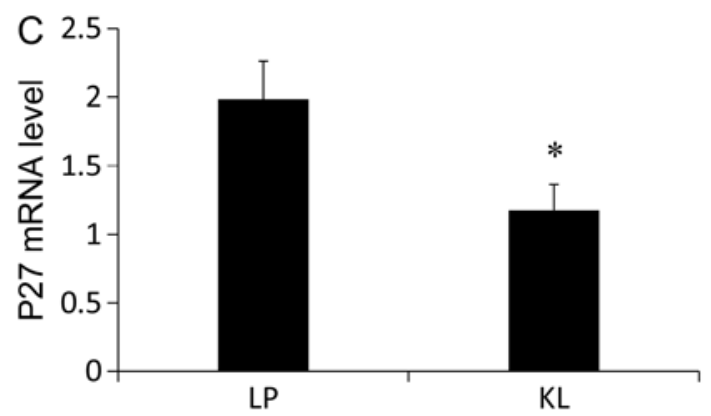

Figure 6. Effects of KL on hepatocyte proliferation. (A) Immunofluorescence of the percentage of Ki67-labeled nuclei of the KL and LP groups ( $\mathrm{n}=6$, original magnification, x200). Sections of liver were stained with DAPI (blue) and counterstaining with anti-Ki67 (red). (B) The mRNA expression levels of P21 and (C) P27 were indicated. Data represent the mean \pm standard error of the mean. " $\mathrm{P}<0.05$ vs. LP group. LP, liver perfusion; KL, kidney liver perfusion.

A

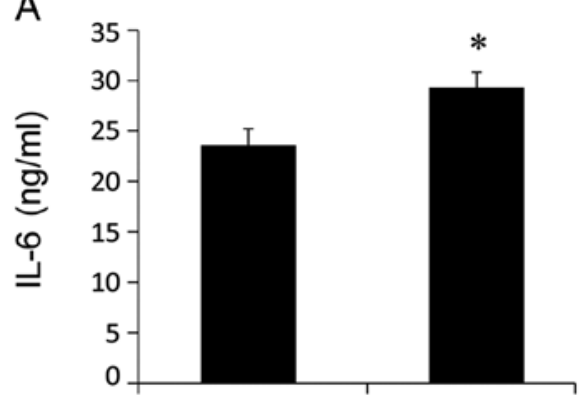

LP

C

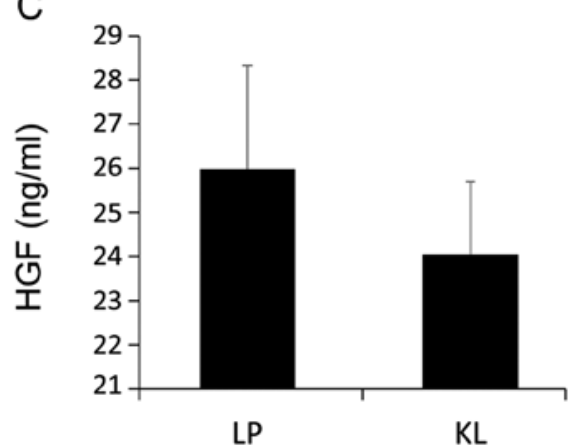

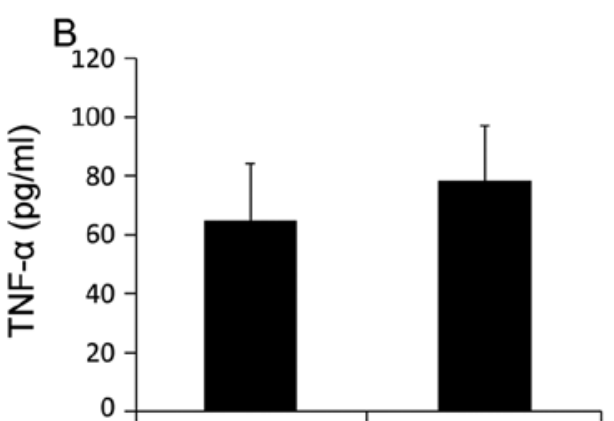

LP

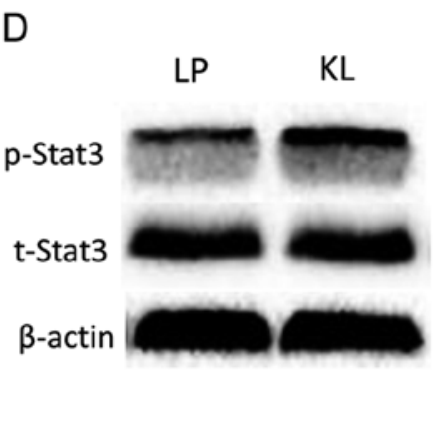

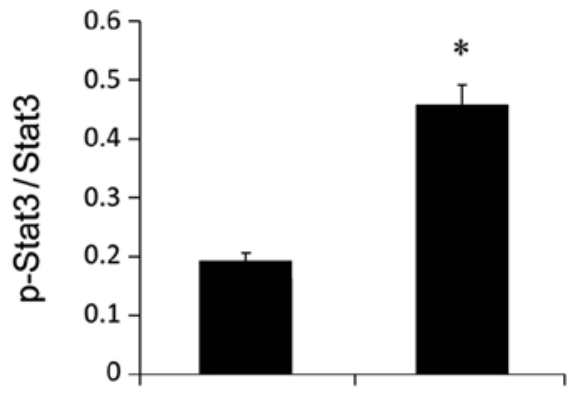

LP

$\mathrm{KL}$

Figure 7. Effects of KL on hepatocyte proliferation signaling. (A) IL-6, (B) HGF and (C) TNF- $\alpha$ were analyzed at the end of perfusion. (D) Protein expression and quantitative data of $\mathrm{p}-\mathrm{Stat} 3 / \mathrm{t}-\mathrm{Stat} 3$ were indicated. Data represent the mean \pm standard error of the mean. " $\mathrm{P}<0.05$ vs. LP group. IL, interleukin; HGF, hepatocyte growth factor; TNF, tumor necrosis factor; LP, liver perfusion; KL, kidney liver perfusion; p-stat3, phosphorylated-signal transducer and activator of transcription; $\mathrm{t}$, total.

\section{Discussion}

In the present study, overall $\mathrm{pH}$ and $\mathrm{K}^{+}$values of the $\mathrm{KL}$ group were different compared with the LP group and more stable. ALT, AST and LDH levels increased progressively over time in the LP group and were different at different time points compared with pre-perfusion levels and the KL group. The results indicated that KL group was superior to the LP group. KL reduced portal VR and was associated with lower ATP consumption compared with the LP group. The results also suggested the lack of involvement of hepatocyte apoptosis. In addition, for the first time to the best of our knowledge liver 
A

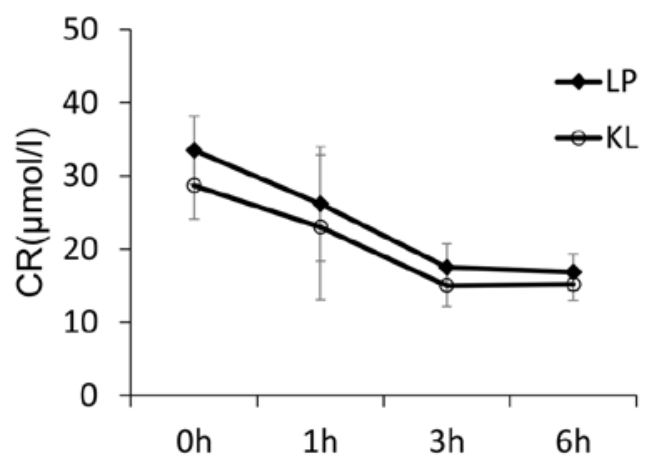

B

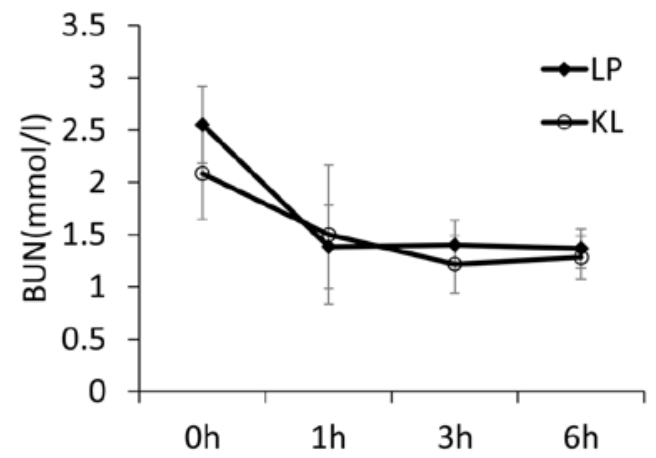

Figure 8. Kidney function tests were indicated over time. (A) CR amd (B) BUN were analyzed over time. Circles or boxes represented the mean values whereas the bars represented the standard error of mean. BUN, blood urea nitrogen; CR, creatinine.

proliferation was identified to be upregulated in KL compared with LP ex vivo.

Over the past decade, the number of liver transplants has been relatively stagnant. Notably, the requirement for surgery has expanded; however, there are few liver grafts available. Therefore, there is an urgent need to identify an effective way to increase the viability and usability of marginal grafts (DCD and ECD livers). MP has been proposed to be a beneficial alternative to SCS, particularly for marginal grafts.

Several studies have indicated that the use of HMP is associated with improved liver function and minimized liver damage $(1,2)$. However, due to the absence of homeostatic organs, the persistence of the urea cycle and the high insulin resistance to glycogenolytic leads to a steady increase in circulating urea and glucose levels, which contributes to the steady rise of osmotic pressure in hepatic cells (14). The more important metabolites, hydrogen ions and electrolytes, also tend to accumulate, which leads to acid-base and electrolyte balance alterations during circulation on perfusion, despite the efforts to offset these effects with the use of counteracting substances (including bicarbonate) to control the biochemical environment, which unfortunately leads to further deviations from normal physiology (15). Previous studies have demonstrated that the kidneys synchronously participate with the liver as a natural filter of waste that accumulates during perfusion, purging the circuit and avoiding the accumulation of counteracting substances $(6,7)$. In order to improve acid-base and electrolyte balance alterations that occur during perfusion, and possibly affect organ survival and function, a second organ-kidney with a homeostatic function was added to the circulation. Compared with previous studies, the $\mathrm{pH}$ value and $\mathrm{K}^{+}$levels of the KL group were identified to be more linear and the levels were more similar over time compared with the LP group, which suggested more stable circulation conditions within the KL group. The liver lacks any organs that control homeostatic $\mathrm{pH}$ (kidneys or lungs) during MP and therefore complex acid-base alterations have not yet been compensated (16). In the KL group, a functional kidney ensures that excess $\mathrm{HCO}_{3}^{-}$is excreted into the urine. In the present study, this was suggested by the flatter appearance of the $\mathrm{pH}$ curve compared with the LP group. Similarly, the levels of $\mathrm{K}^{+}$in the KL group were maintained at a greater stability compared with the separate liver circuits. However, it remains uncertain whether a more physiological environment can translate into a better-preserved organ.

Based on the effects that were observed on the $\mathrm{pH}$ value and $\mathrm{K}^{+}$levels during MP, whether KL-induced physiological milieu participated in the protection against liver damage induced by ischemic injury was next examined. He et al (17) demonstrated improved hepatocyte and biliary epithelial preservation by liver-kidney normothermic machine perfusion (NMP) compared with liver NMP alone using hematoxylin and eosin, and TUNEL staining. In addition, liver function tests during liver preservation following reperfusion or transplantation revealed that improved liver function was observed in liver-kidney NMP compared with liver NMP alone (18). In agreement with this study, it was demonstrated that ALT, AST and LDH levels increased progressively over time in the LP group and were different at different time points compared with pre-perfusion levels and the KL group. Furthermore, it was also indicated that KL was superior to LP regarding VR and ATP levels. Lack of ATP and direct inhibition at low temperatures can impair the function of $\mathrm{Na}^{+}-\mathrm{K}^{+}$pumps, which are key components involved in preventing cell swelling. The restored ATP content has been indicated to be a good indicator of mitochondrial respiratory function and is associated with the reduction of oxidative stress. Furthermore, the poor recovery of ATP is associated with poor liver function (18). In the present study, KL reduced VR efficiently, which was associated with lower ATP consumption compared with LP. This may be due to its physiological milieu.

It was speculated that the KL-induced physiological milieu may lower liver apoptosis compared with the LP group. A TUNEL assay was used to confirm the extent of apoptosis of various cell types by detecting late events, in which major DNA fragmentation occurred (19). The results of the present study demonstrated that no significant difference was identified in the percentage of the TUNEL ${ }^{+}$cells. Notably, apoptosis is triggered by the pro-apoptotic protein Bax, which is transported from the cytoplasm to the mitochondria and is induced by cytochrome $\mathrm{c}$. This promotes the activation of procaspase- 9 and -3 (20).

The caspase family serves an important role in apoptosis. These factors are associated with identifying and determining apoptosis in cells. Caspase-3 is particularly important because it is involved in intrinsic and extrinsic apoptotic pathways (21). In the present study, the mRNA and expression levels of Bax and caspase- 3 were measured, which are commonly used 
targets for detecting apoptosis. The results of the present study confirmed no significant difference was indicated.

Since apoptosis could not explain the protective effect of KL, KL-regulated hepatocyte proliferation response and cell cycle regulators was assessed. Liver regeneration was evaluated using $\mathrm{Ki} 67$ staining. The number of $\mathrm{Ki} 67^{+}$hepatocytes was increased in the KL group compared with the LP group. Furthermore, the expression levels of P21 and P27, which are known cell cycle inhibitors were measured. The mRNA expression levels of P21 and P27 were reduced in the KL group compared with the LP group. The results indicated that KL induced regeneration, not apoptosis and exhibited a better protective effect against liver injury compared with LP.

TNF- $\alpha$ and IL- 6 are the major cytokines that can trigger normally quiescent hepatocytes to enter cell cycle arrest due to liver injury (22). HGF, which is synthesized in response to IL-6 in the liver, is also a key factor for liver growth and function (23). Examination of cytokine production in the liver revealed that the IL-6 levels under combined perfusion were raised compared with liver perfusion alone. However, no significant changes of HGF and TNF- $\alpha$ were observed between the two groups. Notably, IL-6 stimulates compensatory hepatocyte proliferation by activating Stat3, which serves a key role in hepatocyte proliferation during liver regenerative responses (24). Accordingly, the protein expression levels of Stat 3 were measured. The present results revealed the same trend as indicated for the IL-6 levels. These results suggested that $\mathrm{KL}$ induced liver regeneration by upregulating the IL-6/Stat3 signaling pathway.

There are several limitations including the lack of a control group-SCS, which was demonstrated in the authors' previous study that HMP is superior to SCS in maintaining the architecture and function of liver grafts (2), and the lack of blood coagulation and bilirubin measurements to assess liver function. Further future experiments validating the participation of the proposed signaling pathway is also needed. Nevertheless, this study provides clear clues for future studies.

In conclusion, different metabolites tend to accumulate during circulation on perfusion, despite the efforts to offset by use of counteracting substances to control the biochemical environment. Previously, researchers used the membrane dialyzers to filter these metabolites. Then the hypothesis that a filter organ can better improve perfusion blood biochemistry has been identified by adding a kidney to the ex vivo liver perfusion. Adding this filter organ to the liver circulation helps maintain a better physiological balance in the novel multi-organ perfusion model, which overcomes the technical challenges of extracorporeal perfusion of multiple organs and the analysis of the response of two organs to the stress states. To the best of our knowledge this study revealed for the first time that combined KL HMP provided a more proactive repair capability by maintaining liver regeneration via upregulation of the IL-6/Stat3 signaling pathway. Accordingly, this KL HMP model could be a potentially important strategy for biochemical reconditioning of the grafts and may be potentially applicable in clinical practice.

\section{Acknowledgements}

Not applicable.

\section{Funding}

The present study was supported by the National Natural Science Foundation of China (grant nos. 81421062 and 81470891); the Science and Technology Bureau of Zhejiang Province, China (grant no.2016C33145); the 863 National High Technology Research and Development Program of China for young scientist (grant no. 2015AA020923); the Public Technology Research Projects (grant no. LGF18C100001); and the China Postdoctoral Science Foundation (grant no. 2017M610374).

\section{Availability of data and materials}

All data generated and analyzed during the present study are included in this published article.

\section{Authors' contributions}

JL, JJ, NH, LZ and SZ contributed in the protocol design; experimental design and implementation; manuscript drafting; critical revisions of manuscript final approval of manuscript. LJ, HY, HL and HX contributed to the conception; critical revisions of manuscript; funding securement; and final approval of manuscript. NH, YP and HX contributed in the data acquisition; data analysis and statistics; data interpretation; final approval of manuscript; and funding securement.

\section{Ethics approval and consent to participate}

All experimental procedures in the present study were approved by the Ethics Committee of The First Affiliated Hospital, College of Medicine, Zhejiang University (2016-374) and were implemented in accordance with the Animal Research: Reporting In Vivo Experiments guidelines (http://www.nc3rs. org/ARRIVE) and the AVMA euthanasia guidelines 2013.

\section{Patient consent for publication}

Not applicable.

\section{Competing interests}

The authors declare that they have no competing interests and all authors confirm its accuracy.

\section{References}

1. Schlegel A and Dutkowski P: Role of hypothermic machine perfusion in liver transplantation. Transpl Int 28: 677-689, 2015.

2. Jia JJ, Zhang J, Li JH, Chen XD, Jiang L, Zhou YF, He N, Xie HY, Zhou L and Zheng SS: Influence of perfusate on liver viability during hypothermic machine perfusion. World J Gastroenterol 21: 8848-8857, 2015.

3. Hosgood SA, Bagul A and Nicholson ML: Minimising cold ischaemic injury in an experimental model of kidney transplantation. Eur J Clin Invest 41: 233-240, 2011.

4. Newsome PN, Henderson NC, Nelson LJ, Dabos C, Filippi C, Bellamy C, Howie F, Clutton RE, King T, Lee A, et al: Development of an invasively monitored porcine model of acetaminophen-induced acute liver failure. BMC Gastroenterol 10: 34, 2010.

5. Wyss $M$ and Kaddurah-Daouk R: Creatine and creatinine metabolism. Physiol Rev 80: 1107-1213, 2000. 
6. Chung WY, Gravante G, Al-Leswas D, Arshad A, Sorge R, Watson CC, Pollard C, Metcalfe MS and Dennison AR: The development of a multiorgan ex vivo perfused model: Results with the porcine liver-kidney circuit over $24 \mathrm{~h}$. Artif Organs 37: 457-466, 2013

7. Chung WY, Gravante G, Al-Leswas D, Alzaraa A, Sorge R, Ong SL, Pollard C, Lloyd DM, Metcalfe MS and Dennison AR: The autologous normothermic ex vivo perfused porcine liver-kidney model: Improving the circuit's biochemical and acid-base environment. Am J Surg 204: 518-526, 2012.

8. Mahboub P, Ottens P, Seelen M, t Hart N, Van Goor H, Ploeg R, Martins PN and Leuvenink H: Gradual rewarming with gradual increase in pressure during machine perfusion after cold static preservation reduces kidney ischemia reperfusion injury. PLoS One 10: e0143859, 2015.

9. Kamada N and Calne RY: A surgical experience with five hundred thirty liver transplants in the rat. Surgery 93: 64-69, 1983.

10. Lai SS,Zhao DD, Cao P, Lu K, Luo OY, Chen WB, Liu J, Jiang EZ $\mathrm{Yu} \mathrm{ZH}, \mathrm{Lee} \mathrm{G}$, et al: PP2Aco positively regulates the termination of liver regeneration in mice through the AKT/GSK3 $\beta /$ Cyclin D1 pathway. J Hepatol 64: 352-360, 2016.

11. Huang Q, Zhan L, Cao H, Li J, Lyu Y, Guo X, Zhang J, Ji L, Ren T, An J, et al: Increased mitochondrial fission promotes autophagy and hepatocellular carcinoma cell survival through the ROS-modulated coordinated regulation of the NFKB and TP53 pathways. Autophagy 12: 999-1014, 2016.

12. Livak KJ and Schmittgen TD: Analysis of relative gene expression data using real-time quantitative PCR and the 2(-Delta Delta C(T)) method. Methods 25: 402-408, 2001

13. Yang $\mathrm{X}$, Jiang $\mathrm{H}$ and Shi Y: Upregulation of heme oxygenase-1 expression by curcumin conferring protection from hydrogen peroxide-induced apoptosis in $\mathrm{H} 9 \mathrm{c} 2$ cardiomyoblasts. Cell Biosci 7: 20, 2017.

14. Reiling J,Lockwood DS, Simpson AH, Campbell CM, Bridle KR, Santrampurwala N, Britton LJ, Crawford DH, Dejong CH and Fawcett J: Urea production during normothermic machine perfusion: Price of success? Liver Transpl 21: 700-703, 2015.

15. Chung WY, Gravante G, Eltweri A, Sorge R, Ong SL, Pollard C, Metcalfe M and Dennison A: The 'kidney-liver' multiorgan ex vivo perfused model improves the circuit's biochemical milieu during perfusion compared to the 'liver-kidney' counterpart. J Artif Organs 18: 151-161, 2015.

16. Gravante G, Ong SL, Metcalfe MS, Sorge R, Fox AJ, Lloyd DM, Maddern GJ and Dennison AR: Changes in acid-base balance during electrolytic ablation in an ex vivo perfused liver model. Am J Surg 204: 666-670, 2012.
17. He X, Ji F, Zhang Z, Tang Y, Yang L, Huang S, Li W, Su Q, Xiong W,Zhu Z, et al: Combined liver-kidney perfusion enhances protective effects of normothermic perfusion on liver grafts from donation after cardiac death. Liver Transpl 24: 67-79, 2018.

18. Liu Q, Nassar A, Farias K, Buccini L, Baldwin W, Mangino M, Bennett A, O'Rourke C, Okamoto T, Uso TD, et al: Sanguineous normothermic machine perfusion improves hemodynamics and biliary epithelial regeneration in donation after cardiac death porcine livers. Liver Transpl 20: 987-999, 2014

19. Boncompagni E, Gini E, Ferrigno A, Milanesi G, Gringeri E, Barni S, Cillo U, Vairetti M and Freitas I: Decreased apoptosis in fatty livers submitted to subnormothermic machine-perfusion respect to cold storage. Eur J Histochem 55: e40, 2011.

20. Guan JJ, Zhang XD, Sun W, Qi L, Wu JC and Qin ZH: DRAM1 regulates apoptosis through increasing protein levels and lysosomal localization of BAX. Cell Death Dis 6: e1624, 2015.

21. Chen H, Zhang J, Gao Y, Liu S, Koh K, Zhu X and Yin Y: Sensitive cell apoptosis assay based on caspase-3 activity detection with graphene oxide-assisted electrochemical signal amplification. Biosens Bioelectron 68: 777-782, 2015.

22. López-Luque J, Caballero-Diaz D, Martinez-Palacián A, Roncero C, Moreno-Càceres J, García-Bravo M, Grueso E, Fernández A, Crosas-Molist E, García-Álvaro M, et al: Dissecting the role of epidermal growth factor receptor catalytic activity during liver regeneration and hepatocarcinogenesis. Hepatology 63: 604-619, 2016.

23. Lai SS, Zhao DD, Cao P, Lu K, Luo OY, Chen WB, Liu J, Jiang EZ, $\mathrm{Yu} Z \mathrm{ZH}, \mathrm{Lee} \mathrm{G}$, et al: $\mathrm{PP} 2 \mathrm{Ac} \alpha$ positively regulates the termination of liver regeneration in mice through the AKT/GSK3 $3 /$ Cyclin D1 pathway. J Hepatol 64: 352-360, 2016.

24. Liu Y, Shao M, Wu Y, Yan C, Jiang S, Liu J, Dai J, Yang L, Li J, Jia W, et al: Role for the endoplasmic reticulum stress sensor IRE1 $\alpha$ in liver regenerative responses. J Hepatol 62: 590-598, 2015.

This work is licensed under a Creative Commons Attribution-NonCommercial-NoDerivatives 4.0 International (CC BY-NC-ND 4.0) License. 